\title{
Para Além do QI: Avaliação do Comportamento Adaptativo na Deficiência Intelectual
}

\author{
Silvia Cristina Marceliano Hallberg $\left(\mathbb{D}\right.$, Denise Ruschel Bandeira ${ }^{1}$ D \\ Universidade Federal do Rio Grande do Sul, Porto Alegre-RS, Brasil
}

\section{RESUMO}

A Deficiência Intelectual (DI) é caracterizada por limitações de inteligência e comportamento adaptativo, que se originam no período desenvolvimental. O comportamento adaptativo (CA) representa o conjunto de habilidades conceituais, sociais e práticas que são aprendidas e executadas por pessoas em suas atividades diárias. Este estudo teórico objetiva discutir a importância do exame do CA na avaliação psicológica de pessoas com DI. É abordado o papel desse exame no diagnóstico, na classificação de gravidade e no planejamento de intervenções e são fornecidas orientações sobre procedimentos e ferramentas de avaliação do CA. As considerações finais situam o conhecimento sobre o CA como uma ferramenta indispensável ao psicólogo que trabalha com a avaliação psicológica da DI e aponta a necessidade de pesquisas de adaptação ou construção de medidas de CA validadas para o Brasil.

Palavras-chave: deficiência intelectual; comportamento adaptativo; avaliação psicológica.

\section{ABSTRACT - Beyond IQ: Assessing Adaptive Behavior in Intellectual Disability}

Intellectual Disability (ID) is characterized by limitations of intelligence and adaptive behavior originating in the developmental period. Adaptive behavior represents the set of conceptual, social and practical skills learned and used by people in their daily activities. This theoretical study aims to discuss the importance of examining adaptive behavior in the psychological assessment of people with ID. The role of this examination in the diagnosis, severity classification, and intervention planning is also addressed. Guidance on procedures and tools for assessing adaptive behavior is provided. Final considerations place knowledge about adaptive behavior as an indispensable tool for the psychologist that works evaluating ID and emphasize the need for adaptation or construction of validated adaptive behavior measures for Brazil.

Keywords: intellectual disability; adaptive behavior; psychological assessment.

\section{RESUMEN - Más Allá del Coeficiente Intelectual: Evaluación del Comportamiento Adaptativo en la Discapacidad Intelectual}

La Discapacidad Intelectual (DI) se caracteriza por limitaciones de inteligencia y conducta adaptativa (CA) que se originan en el período de desarrollo. La CA representa el conjunto de habilidades conceptuales, sociales y prácticas que las personas aprenden y realizan en sus actividades diarias. Este estudio teórico tiene como objetivo discutir la importancia de examinar la CA en la evaluación psicológica de las personas con DI. Se discute el papel de este examen en el diagnóstico, la clasificación de la gravedad y la planificación de la intervención; asimismo, se proporciona orientación sobre procedimientos y herramientas para evaluar la conducta adaptativa. Las consideraciones finales sitúan el conocimiento sobre la CA como una herramienta indispensable para el profesional que trabaja con la evaluación psicológica de la DI y señala la necesidad de investigación de la adaptación o construcción de medidas de CA validadas para Brasil.

Palabras clave: discapacidad intelectual; conducta adaptativo; evaluación psicológica.

Mesmo antes do advento dos testes de inteligência, entre os anos 1900, a Deficiência Intelectual (DI) era descrita em termos relacionados ao que hoje é chamado de prejuízos em comportamento adaptativo $(\mathrm{CA})^{2}$. Embora a presença desses prejuízos faça parte da definição operacional da DI desde 1959, historicamente sua medição se mostrou secundária em relação à aferição do funcionamento intelectual para a determinação do transtorno (Tassé et al., 2012).
A inteligência é um dos construtos mais estudados pela Psicologia. Testes de capacidade intelectual já eram utilizados muitas décadas antes do surgimento da primeira escala de prejuízos em CA. Uma vez que o funcionamento intelectual foi inicialmente mais bem compreendido e capaz de ser formalmente medido, tornou-se um ponto de apoio importante no contexto da avaliação da DI (Candeias et al., 2008; Tassé et al., 2016).

${ }^{1}$ Endereço para correspondência: Rua Ramiro Barcelos, 2600, Sala 220, 2ªndar, Santa Cecília, 90035-003, Porto Alegre, RS. Tel.: (51) 3308-5352. E-mail: bandeira@ufrgs.br Artigo derivado da Tese de doutorado da Sílvia Cristina Marceliano Hallberg com orientação da Denise Ruschel Bandeira, defendida em 2021 , no Programa de Pós-Graduação em Psicologia da Universidade Federal do Rio Grande do Sul (UFRGS).

${ }^{2} \mathrm{Na}$ literatura sobre deficiências físicas e intelectuais, há sobreposição dos termos funcionalidade e comportamento ou funcionamento adaptativo, que, com frequência, são usados como sinônimos (Mecca et al., 2015). De igual modo, no presente artigo, todos esses termos serão tratados como equivalentes. 
A solicitação pelo levantamento do Quociente de Inteligência (QI), número que expressa a capacidade intelectual geral, aferida por testes padronizados (Wechsler, 1939), ainda é uma realidade muito presente no cotidiano do psicólogo. Entretanto, a avaliação que se restringe ao mero levantamento da capacidade intelectiva é de pouca valia para fundamentar o diagnóstico e orientar decisões terapêuticas em casos envolvendo suspeita de DI. Para além de medir inteligência, observa-se uma crescente valorização do exame do funcionamento adaptativo no contexto da DI (Mecca et al., 2015).

Este estudo teórico objetiva discutir a importância da investigação do CA na avaliação psicológica de pessoas com DI. Além de abordar o papel desse exame para diagnóstico do transtorno, classificação de sua gravidade e planejamento de intervenções serão fornecidas orientações sobre procedimentos e ferramentas para melhor avaliação do CA.

\section{Caracterizando a Deficiência Intelectual}

A DI é caracterizada por limitações significativas em funcionamento intelectual e em CA, que são originadas durante o período de desenvolvimento (Associação Americana de Psiquiatria [APA], 2014; Schalock et al., 2021; World Health Organization [WHO], 2018). Essas limitações: (a) refletem uma incapacidade ou restrição no desempenho funcional de papéis e tarefas socialmente esperados; (b) representam uma desvantagem substancial para o indivíduo; (c) são influenciadas por variáveis contextuais; e (d) podem ser mitigadas por meio de intervenções, apoios ou por meio da redução de barreiras que impedem oportunidades, equidade e inclusão (Schalock et al., 2021).

A DI possui múltiplas causas, curso variado e manifestações diversas, que impactam a plena funcionalidade do indivíduo ao longo da vida. É frequentemente mal diagnosticada e está relacionada a um acesso precário aos serviços de saúde (APA, 2014; Salvador-Carulla et al., 2011). Sua prevalência varia de 1 a $3 \%$ da população mundial e a incidência tende a ser maior nos países de baixa renda (Harris, 2006; Maulik et al., 2011). A maioria dos casos possui manifestações leves (85\%), seguidos da DI moderada (10\%), severa (4\%) e profunda (1\%) (King et al., 2009).

No Brasil, a ocorrência da DI é estimada em $0,8 \%$ da população. Desse total, mais da metade $(54,8 \%)$ possui grau intenso ou muito intenso de prejuízos funcionais. Somente $30 \%$ dos deficientes intelectuais, no país, frequentam algum serviço de reabilitação em saúde (Instituto Brasileiro de Geografia e Estatística [IBGE], 2015).

\section{Caracterizando o Comportamento Adaptativo e seus Domínios}

O CA representa o "conjunto de habilidades conceituais, sociais e práticas que foram aprendidas e são executadas por pessoas em suas vidas cotidianas" (Schalock et al., 2021, p. 32). Refere-se à eficiência com que o indivíduo toma conta de si e se relaciona com os outros em sua vida diária (Grossman, 1983). Trata-se de "quão bem uma pessoa alcança padrões de sua comunidade em termos de independência pessoal e responsabilidade social em comparação a outros com idade e antecedentes socioculturais similares" (APA, 2014, p. 37). Pode ainda ser definido como o desempenho autossuficiente das atividades diárias que são necessárias para vida pessoal e social (Sparrow et al., 2019). São aquelas tarefas que o indivíduo rotineiramente executa em seu dia a dia, sem avisos, apoios ou lembretes (Saulnier, \& Klaiman, 2018b).

Como principais características do CA, pode-se destacar que ele:
a) é relacionado à idade;
b) possui correlação com funcionamento intelectual;
c) é dependente de contexto;
d) é modificável;
e) é aferido com base no desempenho típico;
f) é composto por três domínios.

O funcionamento adaptativo de uma pessoa não pode ser compreendido dissociado de sua idade. O CA é relacionado às expectativas em termos de funcionalidade para determinandos grupos etários. As habilidades adaptativas típicas de um pré-escolar, por exemplo, são muito diferentes daquelas esperadas em adultos. Além disso, elas tendem a aumentar de complexidade conforme a idade, até atingir um platô na vida adulta. Indivíduos acumulam um repertório maior e mais complexo de capacidades adaptativas à medida que envelhecem. Ainda em relação à idade, desempenhos em escores de CA apresentam maior variabilidade nos indivíduos mais jovens $\mathrm{e}$ maior estabilidade em adultos (Tassé et al., 2018).

Outra característica do CA é sua forte correlação com funcionamento intelectual. Apesar de correlacionados, não existem estudos publicados que apoiem um nexo de causalidade entre inteligência e habilidades adaptativas. Prejuízos em CA não são ocasionados por limitações intelectuais, embora essas condições possam ocorrer concomitantemente (Tassé et al., 2016).

Também o funcionamento adaptativo é dependente de contexto, pois o que é exigido em termos de condutas adaptativas varia conforme aspectos culturais. Assim, limitações em CA são investigadas tendo em vista o ambiente típico dos grupos etários e do grupo cultural da pessoa avaliada (Tassé et al., 2018).

Ainda, o CA é um construto modificável, suscetível a incremento e deterioração. $O$ ganho de experiências e aprendizagens, que o desenvolvimento acarreta, contribuem para o aumento de CA. Intervenções terapêuticas ou a falta delas podem resultar em alterações em funções adaptativas. Mudanças ambientais, como migração, 
hospitalização ou encarceramento, podem afetar as habilidades adaptativas. Igualmente eventos traumáticos, como abuso físico ou emocional, podem alterar níveis de CA (AAIDD, 2015; APA, 2014; Saulnier, \& Klaiman, 2018a; Tassé et al., 2012).

Em contraste com a inteligência, que é aferida a partir do desempenho máximo, o CA é avaliado com base no desempenho típico do indivíduo durante a execução de rotinas diárias e em circunstâncias variáveis. Para a avaliação do desenvolvimento do CA, o examinador precisa saber o que a criança normalmente faz. Se o funcionamento intelectual é entendido como um repertório das capacidades de um indivíduo, o CA é melhor representado como a aplicação funcional dessas capacidades. Essa distinção ajuda a entender, por exemplo, uma questão crítica em autistas com bom rendimento intelectual que, mesmo tendo capacidade intelectiva preservada, não apresentam plena funcionalidade em seu cotidiano para realização de tarefas sociais, práticas, acadêmicas ou profissionais (Harman, \& Smith-Bonahue, 2017; Sparrow et al., 2019; Tassé et al., 2012, 2018).

O CA é composto por três domínios: conceitual, social e prático (APA, 2014; Schalock et al., 2021). O domínio conceitual envolve competências em termos de memória, linguagem, leitura, escrita, raciocínio matemático, aquisição de conhecimentos práticos, solução de problemas e julgamento em situações novas. Também envolve a compreensão de dinheiro, de tempo e de conceitos numéricos (Tassé et al., 2018). Embora o domínio conceitual inclua muitas habilidades cognitivas, o psicólogo não deve confundi-lo com eficiência intelectual. Uma criança pode apresentar QI preservado e mesmo assim manifestar prejuízos na dimensão conceitual.

Nos casos envolvendo DI, os prejuízos em habilidades conceituais podem ser verificados por meio de dificuldades em organizar ou planejar atividades futuras de forma independente, para resolver problemas ou administrar de modo adequado situações complexas, para pensar de modo lógico e abstrato. Há ainda dificuldades para comunicar pensamentos ou ideias complexas e para compreender símbolos sofisticados, como operações matemáticas (Schalock et al., 2012).

O domínio social abarca percepção de pensamentos, sentimentos e experiências dos outros, empatia, habilidades de comunicação interpessoal, habilidades para fazer amizade e julgamento social (APA, 2014). Além de incluir essas habilidades socioemocionais, a dimensão social envolve autoestima, responsabilidade social, credulidade, capacidade para seguir regras, habilidade para evitar ser vítima e resolução de problemas sociais (Tassé et al., 2018).

Problemas em habilidades adaptativas sociais, em casos de DI, são observados por meio de um julgamento social prejudicado e dificuldades para aprender a partir das experiências, especialmente no que diz respeito às interações com outras pessoas. Há maior risco de vitimização devido dificuldades para entender e seguir leis em situações complexas, além de dificuldades para saber em quem confiar. Frequentemente, a pessoa com DI não discrimina as circunstâncias seguras das perigosas. Pode apresentar ingenuidade e sugestionabilidade nas interações com os outros, além de desejo inadequado de agradar figuras de autoridade baseadas na compreensão limitada das situações (Schalock et al., 2012).

O domínio prático compreende capacidade de aprendizagem e autogestão em todos os cenários da vida, como refletido nos cuidados pessoais, responsabilidades acadêmicas ou profissionais, administração do dinheiro, recreação, autocontrole comportamental e organização de tarefas escolares e laborais (APA, 2014). Inclui ainda as habilidades necessárias para manutenção de um ambiente seguro e cuidados com saúde (Tassé et al., 2018).

Nos casos envolvendo DI, os prejuízos no domínio prático comumente se manifestam por meio de dificuldades para realizar as atividades da vida diária de modo independente. É difícil organizar refeições, manter compromissos e manejar medicações de modo seguro. Há comprometimento nas habilidades ocupacionais. Por exemplo, possuem dificuldades para obter um emprego fixo que cubra despesas, para conviver com colegas de trabalho e superiores, para lidar adequadamente com conflitos laborais e para manter um trabalho de alta qualidade sob pressão. Também há limitações na administração do dinheiro. É comum o comportamento de emprestar rendimentos para pessoas que não pagam de volta, passar propriedades ou direitos a outros e fazer compras inconsistentes com o orçamento. Ainda há incapacidades para manutenção de um ambiente seguro. Por exemplo, é comum a falta de precaução com guarda de produtos de limpeza, remédios ou comida e há pouco cuidado na lida com eletricidade, veículos e maquinários (Schalock et al., 2012).

\section{Comportamento Adaptativo e o Diagnóstico da Deficiência Intelectual}

Por definição, há atrasos de funcionamento adaptativo em todos os indivíduos com DI (Saulnier, \& Klaiman, 2018a). Por essa razão, a avaliação psicológica para fins de esclarecimento diagnóstico em casos de suspeita de DI deve incluir o exame de habilidades adaptativas. Tanto a inteligência quanto o CA devem ser avaliados, não havendo qualquer tipo de priorização ou sequência prescrita para essa investigação. Qualquer tentativa de encurtar o processo de investigação de uma possível DI, realizando apenas metade da avaliação necessária é inconsistente com a responsabilidade profissional (Tassé et al., 2016).

Para se obter informações válidas sobre prejuízos em CA, que irão subsidiar o diagnóstico de DI, é necessário o uso de julgamento clínico e de medidas psicométricas adequadas e individualmente administradas. Um diagnóstico válido deve ainda levar em conta a diversidade cultural e linguística, bem como as diferenças nos fatores 
de comunicação, sensoriais, motores e comportamentais dos examinandos (APA, 2014; Schalock et al., 2021).

Prejuízos significativos em CA são determinados quando pelo menos um de seus domínios (conceitual, social, prático) está suficientemente prejudicado a ponto de ser necessário apoio contínuo para que o examinando tenha desempenho adequado em um ou mais contextos, tais como escola, local de trabalho ou em casa. Esse critério é operacionalizado na ocorrência de um desempenho que é aproximadamente dois desvios padrão abaixo da média em um dos três domínios de CA (conceitual, social ou prático) ou numa pontuação geral de uma medida padronizada de funcionamento adaptativo. Além disso, na interpretação desses escores, assim como na aferição de qualquer outra medida em Psicologia, o avaliador deve atentar para o erro padrão de mensuração e limitações dos instrumentos (APA, 2014; Schalock et al., 2021; WHO, 2018).

\section{Comportamento Adaptativo e a Classificação da Deficiência Intelectual}

Uma vez estabelecido o diagnóstico, pode-se especificar a gravidade da DI. Esse processo de classificação está a serviço de levantar mais informações sobre o caso avaliado e embasar, da forma mais completa possível, as decisões necessárias à terapêutica e incremento da funcionalidade (Schalock, \& Luckasson, 2015). O DSM-5 e a CID-11 classificam a DI conforme a sua gravidade em leve, moderada, grave e profunda. Esses especificadores são definidos com base no $\mathrm{CA}$ e não mais em escores de QI (APA, 2014; Schalock et al., 2021; WHO, 2018).

A classificação leve denota dificuldades na aquisição e compreensão de conceitos complexos de linguagem e de educação formal. A maioria das pessoas nessa categoria consegue executar bem atividades domésticas básicas e de autocuidado. Frequentemente conseguem manter um emprego e um modo de vida relativamente independente. Por sua vez, indivíduos afetados por DI de classificação moderada apresentam linguagem e capacidade para aquisição de habilidades acadêmicas mais limitadas. Alguns podem dominar as atividades domésticas básicas e de autocuidado, mas a maioria requer um apoio considerável e consistente para conseguir uma vida independente e trabalho (APA, 2014; WHO, 2018).

A DI de classificação grave cursa com linguagem e capacidade para aquisição de habilidades acadêmicas muito prejudicadas. Geralmente há co-ocorrência de deficiências motoras e necessidade de apoio diário em um ambiente supervisionado para um cuidado adequado. Com treinamento intensivo, é possível a aquisição de habilidades básicas de autocuidado. Já os indivíduos afetados por DI de classificação profunda possuem comunicação muito limitada e capacidade para aprendizado formal restrita a conhecimentos mais básicos e concretos. É comum a presença de deficiências motoras e sensoriais. Normalmente, requerem suporte diário em um ambiente supervisionado para um cuidado adequado. Os casos de nível grave e profundo são distinguidos exclusivamente com base nas diferenças de CA porque medidas de QI são menos válidas na extremidade mais inferior da variação desse coeficiente (APA, 2014; WHO, 2018).

\section{Comportamento Adaptativo e o Mapeamento de Áreas que Necessitam de Intervenções}

A avaliação psicóloga no contexto da DI, além de frequentemente se restringir ao exame do funcionamento intelectual, costuma ser pobre no oferecimento de propostas de ações terapêuticas (Selau, Hallberg, et al., 2020). Como muitas intervenções em DI incluem o desenvolvimento das habilidades necessárias para promover a independência apropriada à idade, a investigação de prejuízos em CA pode auxiliar no planejamento de intervenções voltadas a oportunizar um estilo de vida mais autônomo e funcional para o examinando. Intervenções precoces, individualizadas e continuadas podem incrementar a qualidade de vida e melhorar a apresentação da DI. Mesmo em crianças mais velhas e adultos, o nível de apoio oferecido é capaz de possibilitar a melhoria nas funções adaptativas e na participação em todas as atividades cotidianas (Saulnier, \& Klaiman, 2018c).

A avaliação do CA é capaz de ser usada de forma eficaz para fornecer uma estrutura de apoios, metas para educação e reabilitação (Tassé, 2009). Isso porque uma avaliação abrangente das habilidades adaptativas ajuda a identificar pontos fortes e necessidades, além de focar em metas importantes para programas de intervenção. $\mathrm{O}$ planejamento de intervenções em CA parte do pressuposto de que dentro do funcionamento humano as limitações coexistem com áreas preservadas e pontos fortes (Schalock et al., 2021).

A maioria das pessoas que demonstra déficits significativos em algum dos domínios do CA também exibe bom desempenho em outros (Tassé et al., 2018). Por exemplo, um examinando pode apresentar um adequado desenvolvimento de habilidades conceituais e práticas, mas possuir prejuízos no domínio social. O mapeamento dessas áreas preservadas e prejudicadas é útil para o clínico que deseja desenvolver um perfil dos apoios necessários e priorizar estratégias de intervenção (Schalock et al., 2021, 2012).

Assim, um passo importante para determinar áreas que necessitam de intervenções terapêuticas é conduzir uma aferição apropriada do CA e identificar atrasos ou déficits adaptativos. Além de corresponderem a uma peça-chave no planejamento de ações terapêuticas, medições de CA servem para avaliar o resultado dessas intervenções. A avaliação desses resultados é pertinente para orientar quanto à manutenção ou mudanças de estratégias de apoio (Saulnier, \& Klaiman, 2018a).

\section{Avaliação do Comportamento Adaptativo}

Dado o papel essencial do CA para o diagnóstico, a classificação e o planejamento de intervenções em DI, o conhecimento sobre os procedimentos e instrumentos 
de investigação desse construto é indispensável ao psicólogo que trabalha avaliando esses casos. Nesse contexto, a AAIDD (Schalock et al., 2021) recomenda que a aferição do CA seja fundamentada em três pressupostos. Primeiramente, a avaliação deve ser baseada no desempenho típico. O funcionamento adaptativo não é uma medida de capacidade ou conhecimento, mas sim daquilo que o indivíduo tipicamente faz e do grau de independência na realização dessas habilidades (Schalock et al., 2021; Tassé, 2009). Trata-se daquilo que a pessoa normalmente faz, em vez do que poderia fazer. Em segundo lugar, deve-se procurar mapear tanto as áreas preservadas, quanto as prejudicadas. Limitações em habilidades adaptativas frequentemente coexistem com pontos fortes em indivíduos com DI. Por último, a avaliação deve ser feita tendo em vista os aspectos culturais, o grupo etário do examinando e o apoio individualizado que necessita (Schalock et al., 2021).

A avaliação do CA pode ser realizada por meio de observação direta, entrevistas e uso de medidas individualizadas, culturalmente adaptadas e psicometricamente adequadas (APA, 2014; Saulnier, \& Klaiman, 2018a; Schalock et al., 2021, 2012; WHO, 2018). Além disso, estabelecer um bom relacionamento com o examinando e seus cuidadores diretos, usar múltiplos respondentes e diversas fontes documentais de informação são elementos críticos para garantir a confiabilidade de dados sobre o desenvolvimento do CA (Harrison, \& Oakland, 2003). São exemplos de fontes documentais de informação os registros escolares, os exames médicos, os laudos psicológicos prévios e os relatórios de desempenho laboral (Tassé et al., 2012). Todas essas informações devem ser coletadas de modo a corroborar os dados obtidos por meio da avaliação padronizada (Tassé, 2009).

Os instrumentos padronizados de avaliação do CA podem ser respondidos diretamente ou por meio de um processo de entrevista, com dados fornecidos por múltiplos respondentes. As medidas podem ser empregadas com informantes (pais ou membros da família, professores ou provedor de cuidados) e com o próprio examinando, na medida do possível (APA, 2014; Tassé et al., 2012, 2018). Ressalva-se, entretanto, que medidas administradas por autorrelato não devem ser utilizadas para fins de diagnóstico da DI (Harrison, \& Oakland, 2003; Saulnier, \& Klaiman, 2018a; Tassé et al., 2012). Nesses cenários, é essencial que o respondente seja alguém que conhece bem a pessoa avaliada, incluindo algum familiar, amigo, professor, colega de trabalho, empregador ou profissional envolvido nos cuidados terapêuticos (Tassé et al., 2018). Atualmente, há mais de 30 testes de inteligência disponíveis para uso no Brasil. Diferentemente de instrumentos de avaliação do funcionamento intelectual não há medidas de CA padronizadas para a população brasileira. Isso impossibilita a condução de avaliações com respaldo psicométrico adequado, fazendo com que muitos profissionais optem pelo levantamento de dados mediante entrevistas e julgamento subjetivo. Essa incipiência não somente impacta o diagnóstico, classificação e terapêutica da DI, no país, como também obstaculiza a produção científica nacional sobre o tema. Assim, há importante demanda de estudos de adaptação e de verificação de propriedades psicométricas de medidas de CA no contexto brasileiro (Ferreira et al., 2015; Teixeira et al., 2018).

A Tabela 1 apresenta uma relação, em ordem cronológica de publicação, das medidas de CA mais conhecidas no mundo.

Dos instrumentos relacionados, dois são considerados padrão ouro em aferição de CA: a Vineland Adaptative Behavior Scales- $3^{\circ}$ edição (VABS-III) e o Adaptive Behavior Assessment System-3 ${ }^{\circ}$ edição (ABAS-III) (Teixeira et al., 2018). A VABS-III é administrada individualmente, por meio de uma entrevista. É voltada para medir CA de indivíduos de zero a 90 anos ou mais de idade. Além de avaliar o CA e seus três domínios (conceitual, social e prático), também informa sobre habilidades motoras e comportamento desadaptativo. O tempo de administração varia de acordo com a forma usada, pois possui protocolos de entrevista abrangente (40 a 50 minutos de duração) e de curta aplicação (8 a 10 minutos de duração). Possui também versões específicas para pais e outra para professores (Sparrow et al., 2019). Foi lançada uma tradução recente da VABS-III do inglês para o português. Essa tradução se encontra disponível para uso no Brasil, mas a publicação não abarcou estudos com amostra brasileira. Isso implica que, ao interpretar os resultados, o psicólogo deve ter em mente que está realizando uma comparação de escores entre indivíduos norte-americanos e brasileiros.

O ABAS-III igualmente é administrado individualmente e por meio de uma entrevista. Mede CA de indivíduos de zero a 89 anos de idade. Leva entre 15 e 20 minutos para sua aplicação. Pais, familiares, professores, funcionários de creches, supervisores, conselheiros ou outras pessoas familiarizadas com as atividades diárias da pessoa avaliada podem ser os respondentes. Além disso, o formulário adulto pode ser administrado por autorrelato. O ABAS-III abrange os três domínios adaptativos: conceitual, social e prático. Cada formulário avalia nove ou 10 áreas de habilidades com base na faixa etária. Os itens se concentram em atividades diárias necessárias para atender às demandas ambientais, cuidar de si e interagir com os outros de maneira eficaz e independente (Harrison, \& Oakland, 2015). Apesar de ainda não estar disponível para uso no Brasil, uma adaptação do ABAS-III ao contexto nacional, que incluem estudos com amostras brasileiras, está sendo desenvolvida.

Em processo de publicação, o Inventário Dimensional de Avaliação do Desenvolvimento Infantil (IDADI) avalia atrasos no desenvolvimento de crianças de quatro a 72 meses de idade e também pode ser respondido por professores. Uma das dimensões medidas pelo inventário é o CA e o instrumento possui estudos com amostra brasileira. (Silva et al., 2019). 
Tabela 1

Medidas de Comportamento Adaptativo

\begin{tabular}{|c|c|c|}
\hline Medidas & $\begin{array}{c}\text { Ano de } \\
\text { publicação }\end{array}$ & Idade-alvo \\
\hline Vineland Social Maturity Scale & 1935 & Nascimento à maturidade \\
\hline AAMD* Adaptive Behavior Scale & 1969 & 3 a 17 anos \\
\hline AAMD Adaptive Behavior Scale, Public School Version & 1975 & 1 ano e 3 meses a 13 anos e 2 meses \\
\hline AAMD Adaptive Behavior Scale, School Edition & 1981 & 3 a 18 anos \\
\hline Vineland Adaptive Behavior Scales (VABS) & 1984 & $\begin{array}{l}\text { Nascimento até } 18 \text { anos e } \\
11 \text { meses de idade }\end{array}$ \\
\hline VABS Classroom Edition & 1985 & 3 a 12 anos e 3 meses de idade \\
\hline AAMR $^{* *}$ Adaptive Behavior Scale School:2 & 1993 & 3 a 18 anos \\
\hline Scales od Independent Behavior Scale, Revised (SIB-R) & 1996 & Nascimento até 80 anos \\
\hline Adaptive Behavior Assessment System (ABAS) & 2000 & Nascimento até 89 anos \\
\hline ABAS-II & 2002 & Nascimento até 89 anos \\
\hline VABS-II & 2004 & Nascimento até +90 anos \\
\hline $\begin{array}{l}\text { Adaptive Behavior Evaluation Scale, Revised, } \\
\text { Second Edition (ABES-II) }\end{array}$ & 2006 & 4 a 18 anos \\
\hline ABAS-III & 2015 & Nascimento a 89 anos \\
\hline VABS-III & 2016 & Nascimento a 90 anos ou mais \\
\hline ABES-III & 2017 & 4 a 18 anos \\
\hline Diagnostic Adaptive Behavior Scale (DABS) & 2018 & 4 a 21 anos \\
\hline
\end{tabular}

Fonte. Saulnier e Klaiman (2018a). *Associação Americana de Deficiência Mental. Nome prévio da AAIDD. ** Associação Americana de Retardo Mental. Nome prévio da AAIDD

Outros estudos pertinentes ao tema estão em curso no Brasil. Selau, Silva, et al. (2020) está desenvolvendo a Escala de Funcionamento Adaptativo para Deficiência Intelectual (EFA-DI). Construída para avaliar o CA de indivíduos com DI e entre 7 a 15 anos de idade, a medida se encontra em processo de validação (Selau, Silva, et al., 2020). Hallberg (2019) está adaptando e validando a Diagnostic Adaptive Behavior Scale (DABS). De propriedade da AAIDD, a escala fornece informações confiáveis e válidas sobre limitações em habilidades adaptativas de indivíduos entre 4 a 21 anos de idade. Tanto a EFA-DI quanto a DABS possuem estudos com amostra brasileira.

\section{Considerações Finais}

A inteligência é um construto bastante estudado pela Psicologia. Testes para medi-la estão há muito disponíveis aos profissionais. Se o levantamento do QI é realizado com relativo conforto pela maioria dos psicólogos que trabalham com avaliação psicológica envolvendo a DI, o mesmo não pode ser dito em relação à investigação do funcionamento adaptativo.
Para além da mera aferição da eficiência intelectual, a avaliação psicológica é capaz de oferecer outras informações sobre esses casos. Dados extraídos a partir da investigação do CA são de muita valia para um entendimento aprofundado da funcionalidade da pessoa avaliada. Possuem um papel essencial nos processos de diagnóstico, classificação de gravidade e planejamento de intervenções. Por esse motivo, o conhecimento sobre o CA é uma ferramenta indispensável ao psicólogo que trabalha com a avaliação psicológica da DI.

Essas avaliações, no país, são prejudicadas pela carência de instrumentos padronizados para a população brasileira. Essa lacuna também impacta o desenvolvimento da produção científica nacional sobre CA e DI. Assim, é necessário o investimento de pesquisas de adaptação ou desenvolvimento de instrumentos de CA validados e normatizados para o Brasil. Apesar desse cenário, alguns estudos recentes sobre construção, adaptação e evidências de validade de escalas que avaliam prejuízos em funcionamento adaptativo sinalizam uma perspectiva futura positiva para o aumento do conhecimento sobre CA e para clínica psicológica de pessoas com DI no Brasil. 


\section{Agradecimentos}

Não há menções.

\section{Financiamento}

A presente pesquisa não recebeu nenhuma fonte de financiamento sendo custeada com recursos dos próprios autores.

\section{Contribuições dos autores}

Declaramos que todos os autores participaram da elaboração do manuscrito. Especificamente, o(s) autor(es) Sílvia Cristina Marceliano Hallberg e Denise Ruschel Bandeira participou(ram) da redação inicial do estudo - conceitualização, investigação, visualização, o(s) autor(es) Sílvia Cristina Marceliano Hallberg e Denise Ruschel Bandeira participou(ram) da análise dos dados, e o(s) autor(es) Sílvia Cristina Marceliano Hallberg e Denise Ruschel Bandeira participou(ram) da redação final do trabalho - revisão e edição.

\section{Disponibilidade dos dados e materiais}

Todos os dados e sintaxes gerados e analisados durante esta pesquisa serão tratados com total sigilo devido às exigências do Comitê de Ética em Pesquisa em Seres Humanos. Porém, o conjunto de dados e sintaxes que apoiam as conclusões deste artigo estão disponíveis mediante razoável solicitação ao autor principal do estudo.

\section{Conflito de interesses}

Os autores declaram que não há conflitos de interesses.

\section{Referências}

Associação Americana de Psiquiatria (APA). (2014). Manual Diagnóstico e Estatístico de Transtornos Mentais. (5 ${ }^{\circ}$ ed.), Artmed.

American Association on Intellectual and Developmental Disabilities (AAIDD). (2015, Dezembro 6). Marc Tassé - Adaptative Behavior and the Diagnostic Adaptative Behavior Scale. https://www.youtube.com/watch?v=LmAPmPoeWEQ.

Candeias, A., Almeida, L., Roazzi, A., \& Primi, R. (2008). Inteligência. Definição e medida da confluência de múltiplas concepções. Casa do Psicólogo.

Ferreira, E. J., \& Van Munster, M., A. (2015). Métodos de avaliação do comportamento adaptativo em pessoas com deficiência intelectual: Uma revisão de literatura. Revista Educação Especial, 28(51), 193-208. https://doi.org/10.5902/1984686X.

Grossman, H. J. (1983). Classification in Mental Retardation. American Association on Mental Deficiency.

Hallberg, S. C. M. (2019). Adaptação Transcultural para o Português Brasileiro, Evidências de Validade e Estimativa de Fidedignidade da Diagnostic Adaptive Behavior Scale (DABS) [Tese de Doutorado não publicada]. Universidade Federal do Rio Grande do Sul.

Harman, J. L., \& Smith-Bonahue, T. M. (2017). A Escala de Comportamento Adaptativo Bayley-III. Em L. G. Weiss, T. Oakland \& G. P. Aylward (Orgs.), Bayley-III Uso Clínico e Interpretação (pp.147-166) Pearson Clinical Brasil.

Harris, J. C. (2006). Intellectual Disability Understanding Its Development, Causes, Classification, Evaluation, and Treatment. Oxford University Press.

Harrison, P. L., \& Oakland, T. (2003). Adaptive Behavior Assessment System Second Edition: Manual. Harcourt Assessment.

Harrison, P. L., \& Oakland, T. (2015). Adaptive Behavior Assessment System Third Edition: Manual. WPS.

Instituto Brasileiro de Geografia e Estatística (IBGE). (2015). Pesquisa nacional de saúde 2013: ciclos de vida: Brasil e grandes regiōes. https:// biblioteca.ibge.gov.br/visualizacao/livros/liv94522.pdf.

King, B. H., Toth, K. E., Hodapp, R. M., \& Dykens, E. M. (2009). Intellectual Disability. Em B. J. Sadock, V. A. Sadock, \& P. Ruiz (Orgs.), Comprehensive Textbook of Psychiatry. (9º ed., pp. 3444-3474) Lippincott Williams \& Wilkins.

Maulik, P. K., Mascarenhas, M. N., Mathers, C. D., Dua, T., \& Saxena, S. (2011). Prevalence of intellectual disability: A meta-analysis of population-based studies. Research in Developmental Disabilities, 32(2), 419-436. https://doi.org/10.1016/j.ridd.2010.12.018.

Mecca, T. P., Dias, N. M., Reppold, C. T., Muniz, M., Gomes, C. M. A., Fioravanti-Bastos, A. C. M., Yates, D. B., Carreiro, L. R. R., \& Macedo, E. C. (2015). Funcionamento adaptativo: Panorama nacional e avaliação com o adaptive behavior assessment system. Revista Psicologia: Teoria e Prática, 17(2), 107-122. https://doi.org/10.15348/1980-6906/psicologia.v17n2p107-122.

Salvador-Carulla, L., Reed, G. M., Vaez-Azizi, L. M., Cooper, S., Martinez-Leal, R., Bertelli, M., Adnams, C., Cooray, S., Deb, S., AkouryDirani1, L., Girimaji, S. C., Katz, G., Kwok, H., Luckasson, R., Simeonsson, R., Walsh, C., Munir, K, \&Saxena, S. (2011). Intellectual developmental disorders: Towards a new name, definition and framework for "mental retardation/intellectual disability" in ICD-11. World Psychiatry, 10(3), 175-180. https://doi.org/10.1002/j.2051-5545.2011.tb00045.x.

Saulnier, C. A., \& Klaiman, C. (2018a). Adaptive Behavior Profiles in Intellectual Disability and Genetic Disorders. Em C. A. Saulnier \& C. Klaiman (Orgs.), Essentials of Adaptive Behavior Assessment of Neurodevelopmental Disorders. (pp. 57-78). John Wiley \& Sons.

Saulnier, C. A., \& Klaiman, C. (2018b). Introduction and History of Adaptive Behavior. Em C. A. Saulnier \& C. Klaiman (Orgs.), Essentials of Adaptive Behavior Assessment of Neurodevelopmental Disorders. (pp. 1-16). John Wiley \& Sons.

Saulnier, C. A., \& Klaiman, C. (2018c). The Role of Adaptive Behavior in Treatment and Interventions. Em C. A. Saulnier \& C. Klaiman (Orgs.), Essentials of Adaptive Behavior Assessment of Neurodevelopmental Disorders. (pp. 123-140). John Wiley \& Sons.

Schalock, R. L., Luckasson, R., \& Tassé, M. J. (2021). Intellectual disability: Definition, diagnosis, classification, and systems of supports. (12 ${ }^{\circ}$ ed.), American Association on Intellectual and Development Disabilities.

Schalock, R. L., Luckasson, R., Bradley, V., Buntinx, W., Lachapelle, Y., Shogren, K. A., Snell, M. E., Thompson, J. R., Tasse, M., Verdugo-Alonso, M. A., \& Wehmeyer, M. L. (2012). User's Guide to Accompany the 11th Edition of Intellectual Disability: Definition, Classification, and Systems of Supports. American Association on Intellectual and Development Disabilities.

Schalock, R. L., \& Luckasson, R. (2015). A systematic approach to subgroup classification in intellectual disability. Intellectual and Developmental Disabilities, 53(5), 358-366. https://doi.org/10.1352/1934-9556-53.5.358. 
Selau, T., Silva, M. A., \& Bandeira, D. R. (2020). Construção e evidências de validade de conteúdo da escala de funcionamento adaptativo para deficiência intelectual (EFA-DI). Avaliação Psicológica, 19(3), 333-341. https://doi.org/10.15689/ap.2020.1903.17952.11

Selau, T., Hallberg, S. C. M., \& Bandeira, D. R. (2020, Junho 1-4). Systematic Review and Meta-analysis of Interventions in Adaptive Behavior for Children and Adolescents with Intellectual Disabilities [Apresentação de Pôster]. 144th American Association on Intellectual and Developmental Disabilities (AAIDD) Annual Meeting, Pittsburgh, PA, Estados Unidos da América. https://www. aaidd.org/education/annual-conference/archives/2020-annual-conference.

Silva, M. A., Mendonça Filho, E. J., \& Bandeira, D. R. (2019). Development of the dimensional inventory of child development assessment (IDADI). Psico-USF, 24(1), 11-26. https://doi.org/10.1590/1413-82712019240102.

Sparrow, S. S., Cicchetti, D. V., \& Saulnier, C. A. (2019). Vineland-3 Escalas de Comportamento Adaptativo Víneland-Manual. Pearson Clinical Brasil.

Tassé, M. J. (2009). Adaptive Behavior Assessment and the Diagnosis of Mental Retardation in Capital Cases. Applied Neuropsychology, 16(2), 114-123. https://doi.org/10.1080/09084280902864451.

Tassé, M. J., Luckasson, R., \& Schalock, R. L. (2016). The relation between intellectual functioning and adaptive behavior in the diagnosis of intellectual disability. Intellectual And Developmental Disabilities, 54(6), 381-390. https://doi.org/10.1352/1934-9556-54.6.381.

Tassé, M. J., Schalock, R. L., Balboni, J., Bersani, H. A. Jr, Borthwick-Duffy, S. A., Spreat, S., Thissen, D., Widaman, K. F., \& Zhang, D. (2018). Diagnostic Adaptive Behavior Scale User's Manual. American Association on Intellectual and Development Disabilities.

Tassé, M. J., Schalock, R. L., Balboni, G., Bersani, H., Borthwick-Duffy, S. A., Spreat, S., Thissen, D., Widaman, K. F., \& Zhang, D. (2012). The construct of adaptive behavior: Its conceptualization, measurement, and use in the field of intellectual disability. American Journal on Intellectual and Developmental Disabilities, 117(4), 291-303. https://doi.org/10.1352/1944-7558-117.4.291.

Teixeira, M, C, T, V., Tafla, T. L., Santos, A., \& Carreiro, L, R, R. (2018). Deficiência intelectual: contribuições para sua identificação e avaliação. Em C. A. H. Amato, D. Brunoni \& P. S. Boggio (Orgs.), Distúrbios do desenvolvimento: estudos interdisciplinares. (pp.78-87). Memnon.

Wechsler, D. (1939). The Measurement of adults intelligence. Williams \& Wilkins.

World Health Organization (WHO) (2018). International Classification of Diseases for Mortality and Morbidity Statistics (ICD 11). https://icd.who. int/en.

\section{Sobre as autoras}

Sílvia Cristina Marceliano Hallberg é Psicóloga doutoranda do Programa de Pós-Graduação em Psicologia da Universidade Federal do Rio Grande do Sul (UFRGS).

Denise Ruschel Bandeira é Psicóloga, professora e orientadora do Programa de Pós-Graduação em Psicologia da Universidade Federal do Rio Grande do Sul (UFRGS).

\section{Como citar este artigo}

Hallberg, S. C. M., \& Bandeira, D. R. (2021). Para além do QI: avaliação do comportamento adaptativo na Deficiência Intelectual. Avaliação Psicológica, 20(3), 361-368. http://dx.doi.org/10.15689/ap.2021.2003.19733.10 